\title{
Direct Evidence for a Polar Spot on SV Cam
}

\author{
S.V.Jeffers, A.Collier Cameron J.R.Barnes \\ School of Physics and Astronomy, University of St Andrews, North Haugh, St Andrews, \\ Fife KY16 9SS, U.K. \\ svj1@st-and.ac.uk \\ J.P.Aufdenberg ${ }^{1}$ \\ National Optical Astronomy Observatory, 950 N. Cherry Ave, Tucson, AZ 85726, U.S.A. \\ and \\ G.A.J.Hussain ${ }^{1}$ \\ Astrophysics Div., Research 6 Science Support Department of ESA, ESTEC, Postbus 299, \\ Noordwijk, The Netherlands
}

\begin{abstract}
We have used spectrophotometric data from the Hubble Space Telescope to eclipse-map the primary component of the RS CVn binary SV Cam over 9 HST orbits. We find from these observations and the HIPPARCOS parallax that the surface flux in the eclipsed low-latitude region of the primary is about $30 \%$ lower than computed from a PHOENIX model atmosphere at the effective temperature that best fits the spectral energy distribution of the eclipsed flux. This can only be accounted for if about a third of the primary's surface is covered with unresolved dark star-spots. Extending this to the full surface of the primary, we find that even taking into account this spot filling factor there is an additional flux deficit on the primary star. This can only be explained if there is a large polar spot on the primary star extending from the pole to latitude $48 \pm 6^{\circ}$.

Subject headings: binaries: eclipsing — stars: individual(SV Cam), late-type, activity, spots
\end{abstract}

\footnotetext{
${ }^{1}$ Harvard-Smithsonian Center for Astrophysics, 60 Garden Street, Cambridge, MA 02138 U.S.A.
} 


\section{Introduction}

Over the last 20 years, Doppler imaging studies have revealed that rapidly rotating RS CVn binary systems frequently show long-lived polar caps. These features have been shown to be quite stable over extended time-scales of up to several years. Examples include HR 1099 (Vogt et al. 1999; Petit et al. 2002), EI Eri (Hatzes \& Vogt 1992; Washuettl et al. 2001), UZ Lib (Oláh et al. 2002), HU Vir (Strassmeier 1994; Hatzes 1998), V1355 Ori (Strassmeier 2000) and IL Hya (Weber \& Strassmeier 1998). Theoretical efforts to understand this phenomenon are currently focused on the role of meridional flows in sweeping magnetic flux from decaying active regions up towards the poles (Schrijver \& Title 2001). In order to produce a polar spot in these simulations, however, bipolar active regions have to emerge at a rate 30 times faster than solar, implying that the photospheres of active stars should be peppered with many small spots.

In order to test this idea by measuring this filling factor, we were awarded 9 orbits of HST/STIS time in 2001 November to eclipse-map the starspot distribution on the F9V primary of SV Cam. We obtained spectrophotometric light-curves through three primary eclipses with a photometric signal-to-noise ratio of 5000. At this unprecedented precision, we discovered that using conventional limb-darkening models based on plane-parallel atmospheres gave very poor fits to the primary eclipse profile, particularly around the contact points (Jeffers et al. 2004). We concluded that the spiked pattern of residuals could only be accounted for by a combination of incorrect binary system parameters, and the assumed presence of a polar cap on the primary star.

SV Cam has been the target for numerous spectroscopic and photometric observations. Despite this, there are no established values for the principal stellar parameters that define the shape of the photometric lightcurve i.e. the temperatures and radii of the primary and secondary stars. As shown in Jeffers et al. (2004), the determination of these parameters is essential for a correct modelling of the binary system light-curve. If the system parameters are wrong a satisfactory fit to the lightcurve will only be obtained by placing a greater total spot area than is present when the correct parameters are used. In Jeffers et al. (2004) we determined the radii of the two stars through lightcurve fitting, though it was not possible to model the depth of the eclipse correctly without artificial spot features appearing on the final image. However, when an assumed polar cap was also included in the model, the artificial spot features also disappeared. It is not possible to reconstruct a surface brightness distribution for all latitudes as the poles of SV Cam's primary remain uneclipsed.

In this paper we empirically determine the temperatures for both primary and secondary components by fitting PHOENIX spherical model atmospheres to our spectra. As our HST data is calibrated in absolute flux we determine how much light is missing due to the surface 
of SV Cam's primary being covered with small star-spots. Finally we show that there are two polar caps on the primary star and determine their size.

\section{Observational Details}

Three primary eclipses of SV Cam were observed by the HST, using the Space Telescope Imaging Spectrograph with the G430L grating. The observations comprised 9 spacecraft orbits and spanned 5 days at 2 day intervals from 1-5 November 2001 as shown in Table 1. Summing the recorded counts over the observed wavelength range $2900 \AA$ to $5700 \AA$ for each spectra, results in a photometric lightcurve. A plot showing the 9 spacecraft orbits and the 3 primary eclipses that comprise the photometric lightcurve are shown in Figure 1. The observations have a cadence of 40s and a photometric precision of 0.0002 magnitudes (S:N $5000)$ per $30 \mathrm{~s}$ exposure. These observations and the data reduction method are explained in greater detail in Jeffers et al. (2004).

\section{Phoenix Model Atmospheres}

The synthetic spectra we use in this paper is based on an extension of the grid of PHOENIX model atmospheres described by Allard et al. (2000). This extended grid includes surface gravities, $\log (g)>3.0$ needed for main sequences stars. These models are as described by Hauschildt et al. (1999), but include an updated molecular line list. The models are computed in spherical geometry with full atomic and molecular line blanketing using solar

elemental abundances. In these models, the stellar mass is $0.5 \mathrm{M}_{\odot}$ and the convection treatment assumes a mixing-length to pressure scale height ratio of 2 . There are 117 synthetic spectra in total. The effective temperature runs from $2700 \mathrm{~K}$ to $6500 \mathrm{~K}$ in $100 \mathrm{~K}$ steps at three surface gravities: $\log (g)=4.0,4.5$, and 5.0. The wavelength resolution of these synthetic spectra is $1 \AA$.

The PHOEnIX spectra were converted to the same resolution scale as the HST spectra by convolving them with a Gaussian profile of width equal to the instrumental resolution,

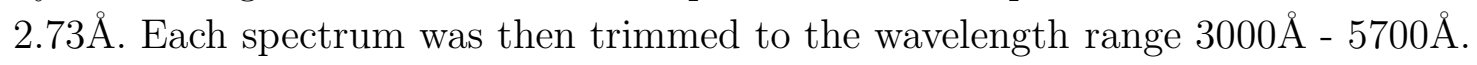

\section{Temperature Fitting using Hipparcos Distance}

In this section we determine the temperature for both the primary and secondary components of SV Cam using PHOEnIX model atmospheres. For consistency we shall denote the 
flux at the Earth by $f$, and the flux at the star by $F$.

\subsection{Primary Star + Secondary Star}

In Jeffers et al. (2004) we accurately determined the radii of the primary and secondary components through lightcurve fitting to be $1.23 \pm 0.03 \mathrm{R}_{\odot}$ and $0.79 \pm 0.02 \mathrm{R}_{\odot}$ for the primary and secondary stars respectively. Knowing these radii we can evaluate the flux contribution from the secondary star relative to that of the primary star. The PHOENIX spectra are scaled by $r^{2} / d_{\text {HIPP }}^{2}$ to give flux at the Earth rather than at the stellar surface. The PHOENIx flux received at the Earth outside of eclipse can be expressed as;

$$
f_{p t o t a l}=\frac{r_{p r i}^{2}}{d_{H I P P}^{2}}\left(F_{p r i}+\frac{r_{s e c}^{2}}{r_{p r i}^{2}} F_{s e c}\right)
$$

where $F_{p r i}$ and $F_{\text {sec }}$ are the PHOENIX model atmosphere fluxes for the primary and secondary stars respectively; $r_{p r i}$ and $r_{s e c}$ are the primary and secondary radii, $d_{H I P P}$ is the HIPPARCOS distance $(84.96 \pm 8.5 \mathrm{pc})$. We fitted this equation for each permutation of primary temperature in the range $T_{p r i}=4500 \mathrm{~K}$ to $6500 \mathrm{~K}$, and secondary temperature $T_{s e c}=$ $2800 \mathrm{~K}$ to 5500 . The best fitting pair of temperatures was determined using $\chi^{2}$ minimisation;

$$
\chi^{2}=\sum_{\lambda(i)=1}^{N}\left(\frac{f_{\text {out }}(i)-\gamma_{\text {total }} * f_{\text {ptotal }}(i)}{\sigma(i)}\right)^{2}
$$

where $f_{\text {out }}$ is an averaged spectrum outside of primary eclipse, and $\gamma_{\text {total }}$ is a scaling factor. A scaling factor is included so that the shape of the spectrum is fitted rather than the absolute flux levels. This scaling factor is defined as;

$$
\gamma_{\text {total }}=\frac{\sum f_{\text {out }} f_{\text {ptotal }} / \sigma^{2}(i)}{\sum\left(f_{\text {ptotal }}\right)^{2} / \sigma^{2}(i)}
$$

The results for the $\chi^{2}$ minimisation are shown in Figure 2 in the form of a contour plot. The minimum $\chi^{2}$ value occurs at $6013 \pm 19 \mathrm{~K}$, and $4804 \pm 143 \mathrm{~K}$, for the primary and secondary stars respectively. As is evident from the shape of the contour plot and the size of the errors for each temperature, the primary temperature is very sensitive, in contrast to the secondary temperature, to small temperature changes (i.e. the secondary places a lower constraint on the solution). The best fitting PHOENIX model atmospheres, $6000 \mathrm{~K}$ and 
$4800 \mathrm{~K}$, and an observed spectrum outside of eclipse are shown in Figure 3. We neglect any reddening correction due to the proximity of SV Cam.

\subsection{Primary Star}

The first step in determining the temperature of the primary star in isolation is to remove its spectrum from that of the secondary star. To achieve this we simply subtracted an average spectrum inside the primary eclipse, $f_{\text {ecl }}$, from an average spectrum outside of eclipse, $f_{\text {out }}$. The spectrum that results is the spectrum of the primary star, but with a reduced flux level that is equivalent to the primary star having a radius of the secondary star. This missing light will be referred to as $f_{m i s}$ in the rest of this paper.

The contribution to the total PHOENIX flux is from only the primary star, so equation ( 1) becomes;

$$
f_{p t o t a l(p r i)}=\frac{r_{p r i}^{2}}{d_{H I P P}^{2}}\left(F_{p r i}\right)
$$

As before, the optimal value of the primary temperature is determined through $\chi^{2}$ minimisation. The scaling factor is then equal to;

$$
\gamma_{p r i}=\frac{\sum f_{\text {mis }} f_{\text {ptotal }(\text { pri })} / \sigma(i)^{2}}{\sum\left(f_{\text {ptotal }(\text { pri })}\right)^{2} / \sigma(i)^{2}}
$$

Figure 4 shows how the reduced $\chi^{2}$ varies with primary temperature. A parabolic fit results in a minimum temperature of $6038 \pm 58 \mathrm{~K}$. The errors were calculated by setting $\Delta \chi^{2}$ $=1$ on the full $\chi^{2}$ value.

\section{Star-spot coverage}

In Figure 5 we show the best fitting PHOENIX spectrum to the primary spectrum, which include a scaling factor. The advantage of using HST spectro-photometric observations is that for each point on our photometric lightcurve we have a value for its absolute flux (i.e. the flux integrated over $3000 \AA$ - $5700 \AA$ in each spectrum). Replotting Figure 5 without the scaling factor in Figure 6, clearly shows that there is a flux deficit on the surface of the primary star, when compared with the flux computed from a PHOENIX model atmosphere at the effective temperature that best fits the spectral energy distribution of the eclipsed flux. 
Using the eclipse mapping technique Jeffers et al. (2004), have already verified the existence of several dark spots at low latitudes on the primary star, but these are insufficient to account for the flux deficit. The total missing flux can only be accounted for if the primary's surface is peppered with unresolved dark star-spots. The dark star-spot filling factor is given by:

$$
\alpha=1-\gamma
$$

where $\alpha$ is the fractional star-spot coverage, and $\gamma$ is the scaling factor as defined in equation 5. This results in the fractional coverage of dark star-spots on SV Cam to be $28 \%$. Taking into account the error on the HIPPARCOS distance this translates to if $\mathrm{d}_{H I P}=93.46 \mathrm{pc}$ then the spot coverage will be $14 \%$, whilst if $\mathrm{d}_{H I P}=76.46 \mathrm{pc}$ then the spot coverage will be $41 \%$.

\section{Polar Spot}

Our determination of the spot coverage factor only accounts for the equatorial latitudes of the primary that are eclipsed by the secondary. However, due to the near $90^{\circ}$ inclination of the binary system it is not possible to recover high-latitude spots or polar caps through the eclipse mapping method. In this section we will firstly show that there is a polar cap on SV Cam and then we will determine its size.

\subsection{Extent of a Polar Cap on SV Cam}

If there is a polar cap on SV Cam, then total flux on the two stars can be expressed as (following from equation( 1$)$ );

$$
f_{p t o t a l}=\frac{r_{p r i}^{2}}{d_{H I P P}^{2}}\left(F_{p r i}\left(1-A_{p c}\right)+\frac{r_{s e c}^{2}}{r_{p r i}^{2}} F_{s e c}\right)
$$

where $\mathrm{A}_{p c}$ is the area of the polar cap. To obtain a full view of the primary star's surface we used an averaged spectrum out of eclipse, but with the secondary's contribution subtracted. It is valid to subtract the PHOENIX spectrum as we assume that the secondary is not spotted or faint enough that the additional flux deficit from star-spots on the secondary is negligible. Following from equation( 3 ) the scaling factor $\gamma$ now becomes: 


$$
\gamma_{p c}=\frac{f_{o u t}-\frac{r_{s e c}^{2}}{d_{H I P P}^{2}} F_{s e c}}{\frac{r_{p r i}^{2}}{d_{H I P P}^{2}}\left(1-A_{p c}\right) F_{p r i}}
$$

If there are no polar caps on SV Cam, $\mathrm{A}_{p c}$ will be zero and the flux deficit should equal the previously determined flux deficit in the primary spectrum, i.e. $\gamma_{p c}=\gamma_{p r i}$. We assume that the previously calculated spot coverage area for the missing light of the primary is valid for the whole primary star, where equation( 5 ) is extended to use $\mathrm{r}_{p r i}$ rather than $\mathrm{r}_{s e c}$. Equivalently we can set $\gamma_{p c}$ equal to $\gamma_{p r i}$ to solve for $\mathrm{A}_{p c}$, e.g.;

$$
\left(1-A_{p c}\right)=\frac{f_{\text {out }}-\frac{r_{\text {sec }}^{2}}{d_{H I P P}^{2}} F_{\text {sec }}}{f_{\text {mis }}}
$$

This equation is illustrated by Figure 7, where the $\gamma_{p c}$ and $\gamma_{p r i}$ are plotted as a function of polar spot area. The intersection point (0.142) indicates the fractional area of the projected disc that is covered by polar caps on SV Cam.

\subsection{Reconstruction of a theoretical polar cap on SV Cam}

The value for $\mathrm{A}_{p c}$ is only a fractional value by which the polar cap has decreased the light of the star. It does not take into account that the star is a sphere, rather than a disc, any limb or gravity darkening, or any spherical oblateness. To account for these parameters, the binary eclipse-mapping tomography code DoTs (Collier Cameron 1997) was used to model theoretical polar spots on the surface of SV Cam. The input to DoTs is in the form of (i) longitude, set to $0^{\circ}$; (ii) latitude, set to $90^{\circ}$; (iii) radius set to $10^{\circ}, 20^{\circ}, 30^{\circ}, 40^{\circ}, 50^{\circ}$ and $60^{\circ}$; (iv) brightness and (v) sharpness, which were both set to make the spot as uniformly dark as possible. Examples of a star with no polar spot, with a $40^{\circ}$ and $60^{\circ}$ polar spot are shown in Figure 8. We assume that the polar cap does not contribute to the total flux of the star.

\subsection{Effect of a polar cap on the primary eclipse}

The effect of the polar cap on the primary eclipse will be to increase the depth of the eclipse. The presence of a polar cap effectively reduces the area of the primary star. When the primary star is then eclipsed the secondary obscures a larger fraction of the primary's surface area than when there was no polar spot. This is illustrated in Figure 9, where there 
is an obvious difference in the eclipse depth for a star with no polar cap and one with a $60^{\circ}$ polar cap.

\subsection{Size of the polar cap on SV Cam}

The fractional decrease in stellar flux as a function of theoretical polar spot size is shown in Figure 10, where we assume that the polar cap contributes negligible flux. Also plotted in Figure 10 is the fractional area of a polar-spot as deduced in the previous section. We conclude that the polar spot on SV Cam is $42 \pm 6^{\circ}$.

\subsection{Lightcurve of SV Cam}

As shown in Figure 9 the presence of a polar spot will increase the depth of the primary eclipse. Figure 12 shows how a $42^{\circ}$ polar cap will influence the lightcurve of SV Cam. Contrasting this, the peppering of small star-spots will decrease the depth of the eclipse as shown in Figure 13. In a complimentary paper, Jeffers et al. (2004), we show how to obtain the best fits to the data by including these effects.

The combination of $42^{\circ}$ polar caps and $28 \%$ surface coverage of small star-spots is shown in Figure 11. This translates to the lightcurve shown in Figure 14. For clarity the lightcurve of SV Cam is also shown without any spot features.

\section{Discussion}

The best fitting temperature of the primary star, $6013 \mathrm{~K}$, is in good agreement with the value of $6000 \mathrm{~K}$ of (Lehmann et al. 2002), whilst the secondary temperature, $4804 \mathrm{~K}$, is in best agreement with the value of $4700 \mathrm{~K}$ of (Rainger et al. 1991). The temperatures that other investigators have observed range from $5700 \mathrm{~K}$ (Patkos \& Hempelmann 1994) to $6440 \mathrm{~K}$ (Albayrak et al. 2001) for the primary star and $4140 \mathrm{~K}$ (Hilditch et al. 1979) to $5600 \mathrm{~K}$ (Popper 1996) for the secondary star. The range in values extends from poor data quality, incomplete lightcurves and the inability to solve simultaneously for the binary system parameters and the spot coverage. However, the advantage that we have is that we have spectrophotometric HST observations i.e. a photometric lightcurve with absolute fluxes

which can be directly compared with PHOENIX model atmospheres to fit for the temperatures of the two stars. 
There are two important results from this paper concerning the spot coverage of SV Cam. The first is that the surface of SV Cam is peppered by $28 \%$ star-spots. Jeffers et al. (2004) reconstructed an image of SV Cam using the HST and ground based photometric data using the eclipse mapping technique. Our final image showed the presence of large dark star-pots at equatorial latitudes but they were not large enough to account for $28 \%$ of the eclipsed part of the primary's surface being covered with star-spots.

The second important result of this paper is that there is certainly a dark polar cap on SV Cam. The existence of polar caps on magnetically active stars has always been a controversial issue. The spectroscopic signature of a polar cap is a line profile with a flat-bottomed core. In some stars with low vsini this could conceivably be caused by chromospheric in-filling of the cores of strong photospheric lines (Byrne 1992, 1996). This result independently confirms that the polar cap is a reality and is not just an artifact of the imaging process. The high resolution spectroscopic observations of Lehmann et al. (2002) showed that SV Cam had no polar cap, but only high latitude spots. The surface area of the primary star that has a polar cap in this work is far greater than in these observations. For the extent of spot coverage that we observe, other Doppler images of RS CVn stars would indicate that this structure is in the form of a polar cap.

In Jeffers et al. (2004) we showed that lightcurve models without a polar cap were not sufficient to correctly fit the primary eclipse profile particularly at the contact points. As shown in figure 9, the presence of a polar cap will increase the depth of the primary eclipse, and if it is not taken into account when fitting the lightcurve, it will not be possible to solve the binary system parameters correctly. Jeffers et al. (2004) further solved for the radii and polar cap size using $\chi^{2}$ minimisation. The optimally fitting polar cap size of $46 \pm 8^{\circ}$ is in good agreement with the value independently determined in this paper.

TiO-band monitoring (O'Neal et al. 1998) has indicated that between $30 \%$ and $50 \%$ of a star's surface may be covered in starpots at all times. Our result of $28 \%$ spot coverage for the eclipsed equatorial regions in addition to a $14 \%$ polar cap are in agreement with TiO-band findings. Conventional Doppler images show that up to $20 \%$ of the star's surface is spotted, mainly in the form of high latitude structure and a polar cap. This discrepancy can be accounted for if the star's surface is peppered with dark star-spots, too small to be resolved with Doppler imaging.

The high total spot coverage on the primary star can have important implications for the overall structure of the star. Spruit \& Weiss (1986) investigated this and concluded that over thermal timescales the star will readjust its structure to compensate in radius and temperature. Also standard colour-surface brightness relation will break down if star-spots cover a large fraction of the stellar surface, but contribute little to the stellar optical flux. 
To illustrate this point, it is possible to determine the stellar radius, $R$, using standard colour-surface brightness relations to obtain the angular diameter and the distance from the HIPPARCos parallax. Using the relation $R \sin i=\left(P_{\text {rot }} / 2 \pi\right) v \operatorname{sini}$ O'Dell et al. (1994), in a survey of axial inclination of $\mathrm{G}$ dwarfs on the $\alpha$ Per and Pleiades clusters found that sini $>1$ for several stars. In the context of our results this can be understood if the high spot coverage causes $R$ to be underestimated, and would account for the large variance of binary system parameters for SV Cam.

This work provides strong evidence, independently of Doppler Imaging, that the poles of SV Cam's primary star are darkened by extensive polar caps, and that at lower latitudes the photosphere is peppered with small star-spots as has been suggested by TiO band monitoring. It is important to establish that these stars are in fact peppered by small starspots in addition to the presence of a polar cap, as this can significantly impact theoretical interpretations of the spot distributions on these stars.

SVJ acknowledges support from a PPARC research studentship and a scholarship from the University of St Andrews.

JPA was funded in part by a Harvard-Smithsonian CfA Postdoctoral Fellowship and in part under contract with the Jet Propulsion Laboratory (JPL) funded by NASA through the Michelson Fellowship Program. JPL is managed for NASA by the California Institute of Technology.

Facilities: HST(STIS).

\section{REFERENCES}

Albayrak B., Demircan O., Djurašević G., Erkapić S., Ak H., 2001, A\&A, 376, 158

Allard F., Hauschildt P. H., Schweitzer A., 2000, ApJ, 539, 366

Byrne P. B., 1992, in NATO ASIC Proc. 375: Sunspots. Theory and Observations Starspots. pp 63-73

Byrne P. B., 1996, in IAU Symposia Vol. 176, On the believability of polar spots. p. 299

Collier Cameron A., 1997, MNRAS, 287, 556

Hatzes A. P., 1998, A\&A, 330, 541

Hatzes A. P., Vogt S. S., 1992, MNRAS, 258, 387 
Hauschildt P. H., Allard F., Ferguson J., Baron E., Alexander D. R., 1999, ApJ, 525, 871

Hilditch R. W., McLean B. J., Harland D. M., 1979, MNRAS, 187, 797

Jeffers S. V., Collier Cameron A., Barnes J. R., Donati J. F., 2004, MNRAS, submitted

Lehmann H., Hempelmann A., Wolter U., 2002, A\&A, 392, 963

O’Dell M. A., Hendry M. A., Collier Cameron A., 1994, MNRAS, 268, 181

Oláh K., Strassmeier K. G., Weber M., 2002, A\&A, 389, 202

O’Neal D., Neff J., Saar S., 1998, ApJ, 507, 919

Patkos L., Hempelmann A., 1994, A\&A, 292, 119

Petit P., Donati J.-F., Collier Cameron A., 2002, MNRAS, 334, 374

Popper D. M., 1996, ApJS, 106, 133

Rainger P. P., Hilditch R. W., Edwin R. P., 1991, MNRAS, 248, 168

Schrijver C. J., Title A. M., 2001, ApJ, 551, 1099

Spruit H. C., Weiss A., 1986, A\&A, 166, 167

Strassmeier K. G., 1994, A\&A, 281, 395

Strassmeier K. G., 2000, A\&A, 357, 608

Vogt S. S., Hatzes A. P., Misch A. A., Kürster M., 1999, ApJS, 121, 547

Washuettl A., Strassmeier K. G., Collier-Cameron A., 2001, in ASP Conf. Ser. 223: 11th Cambridge Workshop on Cool Stars, Stellar Systems and the Sun Latest Doppler images of the RS CVn Binary EI Eridani (CD-ROM Directory: contribs/washuet). pp 1308

Weber M., Strassmeier K. G., 1998, A\&A, 330, 1029

This preprint was prepared with the AAS LATEX macros v5.2. 


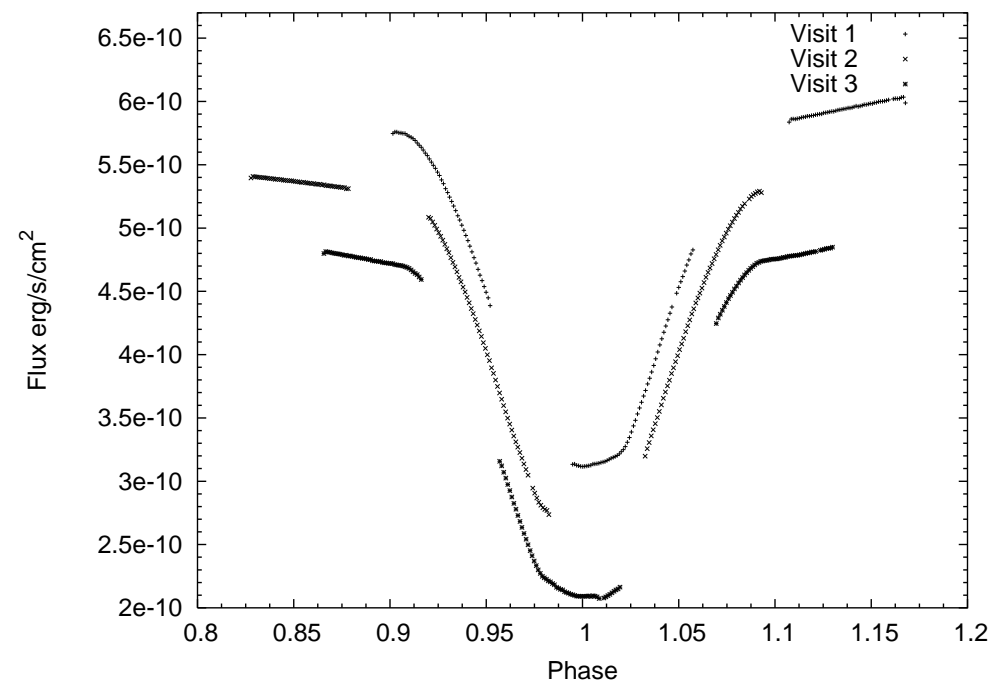

Fig. 1. - The 9 spacecraft orbits that comprise the 3 primary eclipses of SV Cam (where Visit 1 is at the top). An offset of $5 \times 10^{-11}$ has been included for clarity.

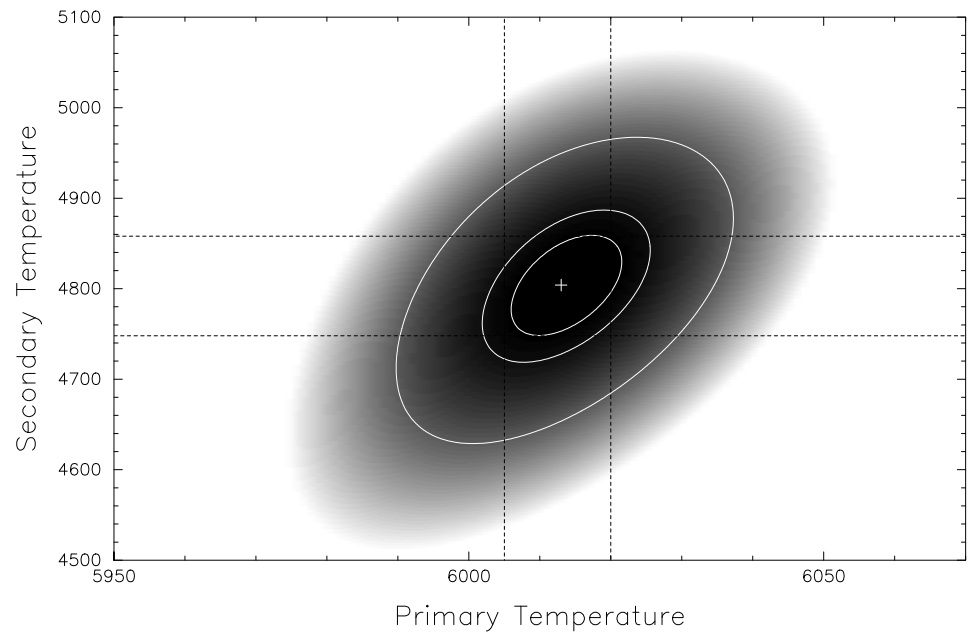

Fig. 2.- Contour plot of the $\chi^{2}$ landscape of the combined primary and secondary stars. The minimum $\chi^{2}$ value occurs at $6013 \pm 19 \mathrm{~K}$, and $4804 \pm 143 \mathrm{~K}$, for the primary and secondary stars respectively. From the centre of the plot the first contour ellipse represents the 1 parameter $1 \sigma$ confidence limit at $63.8 \%$, the second ellipse represents the 2 parameter $1 \sigma$ confidence limit at $63.8 \%$ whilst the third elipse represents the 2 parameter $2.6 \sigma 99 \%$ confidence limit. 


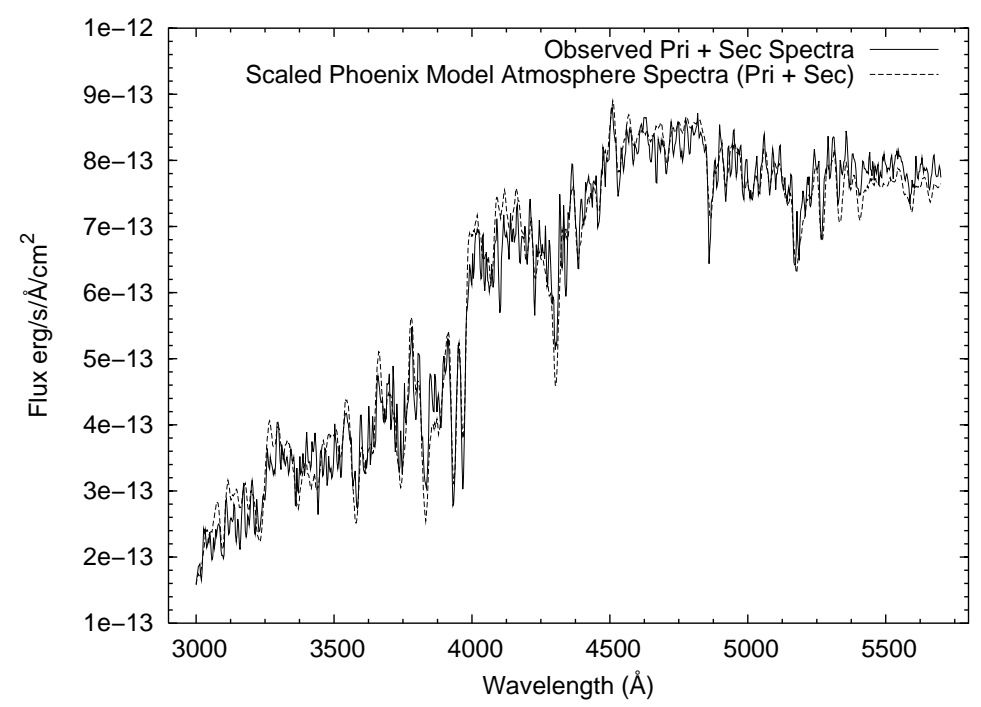

Fig. 3.- Plot showing the combined primary and secondary stars outside of eclipse, $f_{\text {out }}$, and the best fitting PHOENIX spectrum $(6000 \mathrm{~K}$ and $4800 \mathrm{~K}$ for the primary and secondary stars respectively).

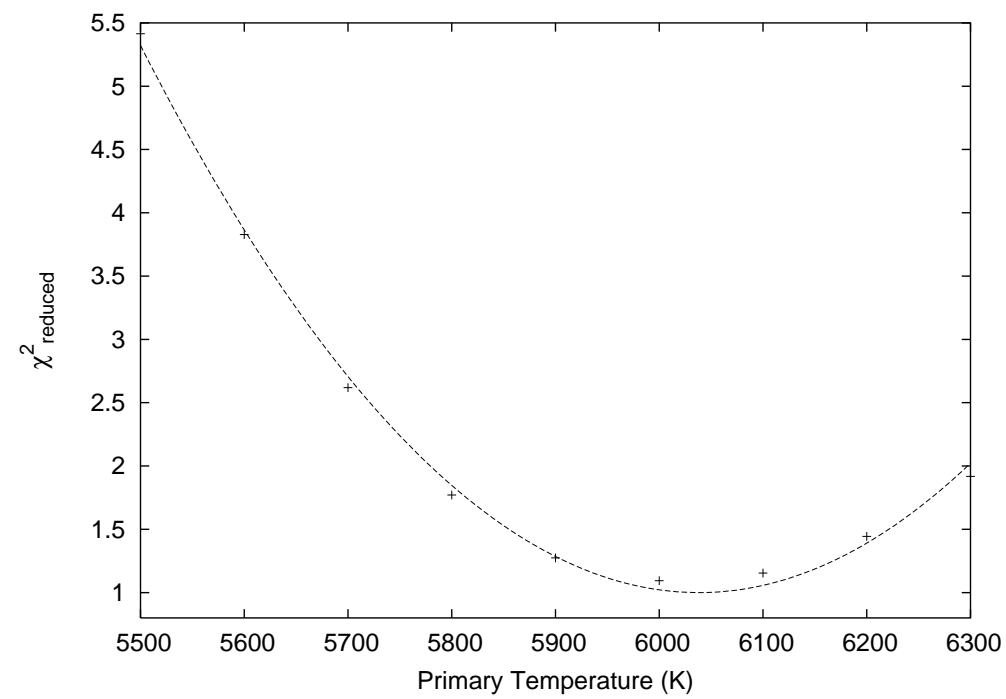

Fig. 4. - The parabolic fit that determines the temperature minimum to be $6038 \pm 58 \mathrm{~K}$ 


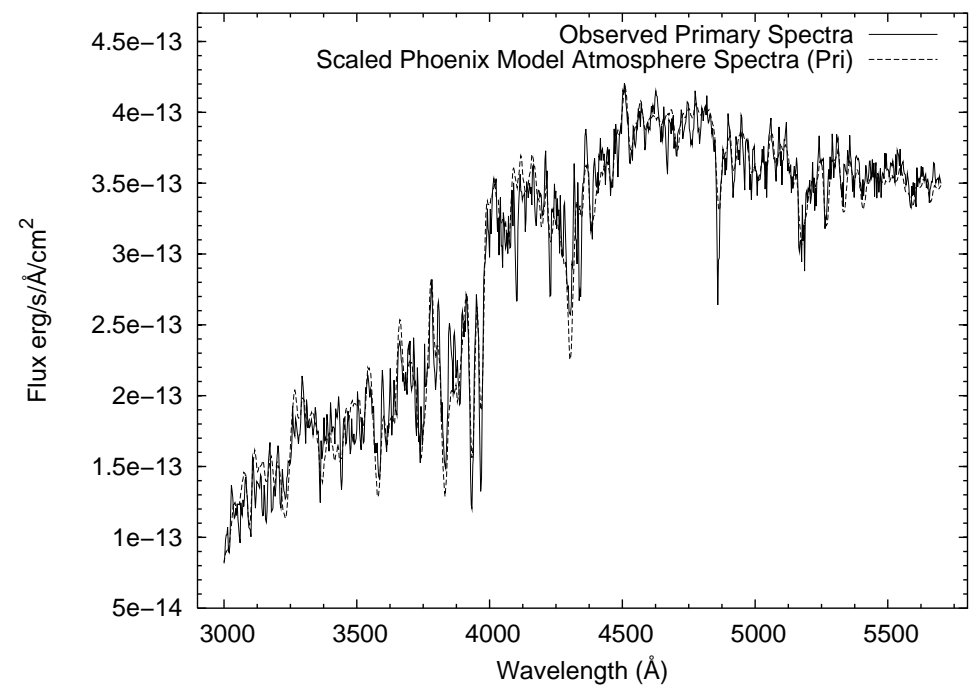

Fig. 5.- Plot showing the spectrum of the primary star, $f_{m i s}$, and the closest match PHOENIX model atmosphere $(6000 \mathrm{~K})$

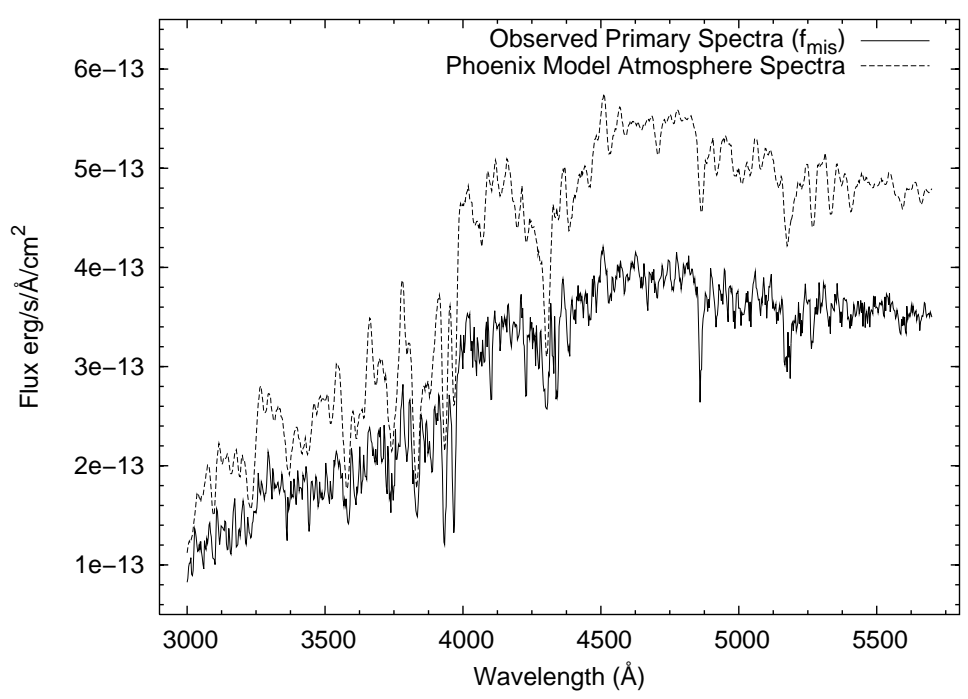

Fig. 6.- As per Figure 5 but without using a scaling factor. This plot illustrates the flux deficit due to the presence of dark star-spots on the surface of the primary. 


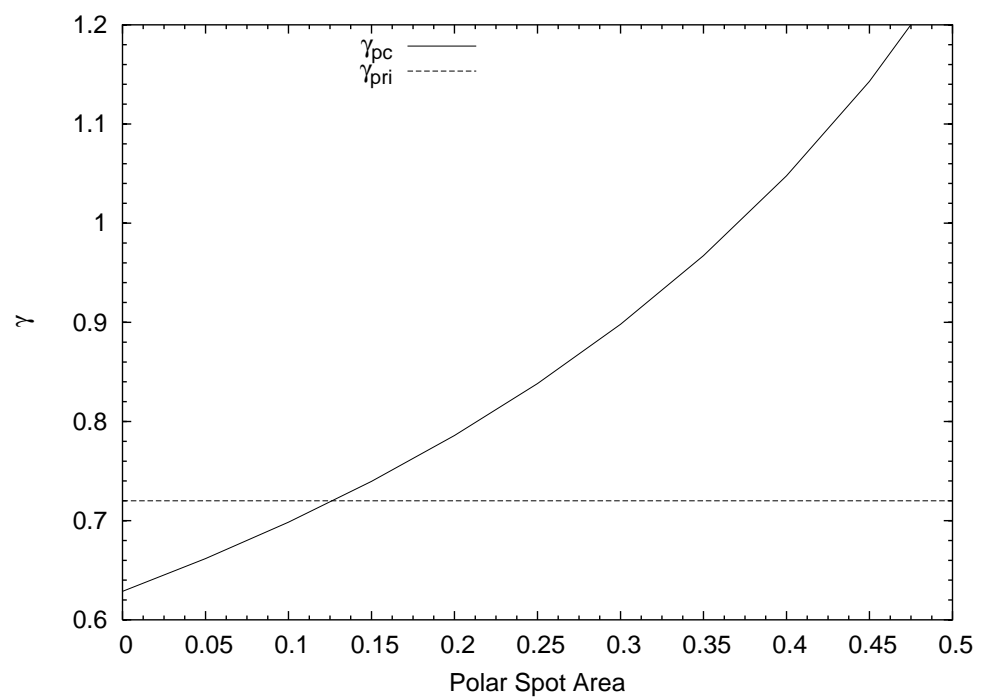

Fig. 7.- Graphical representation of equation 9, where $\gamma_{p c}$ (equation 8) is plotted as a function of polar spot area along with $\gamma_{p r i}$. The area of the polar cap is where the two lines intersect at Spot Area $=0.125$.
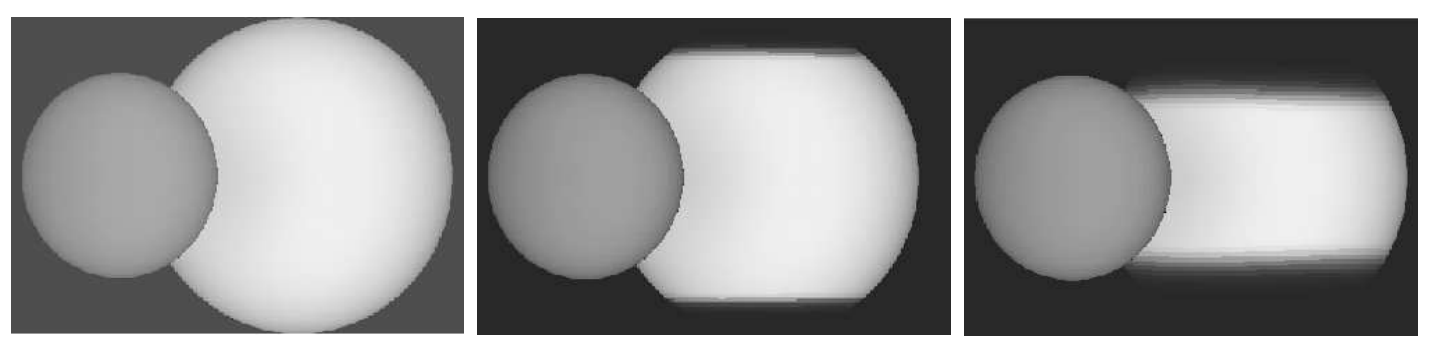

Fig. 8.- Pictorial representation of the polar spots of SV Cam, with no polar cap, a $40^{\circ}$ polar cap and a $60^{\circ}$ polar cap. 


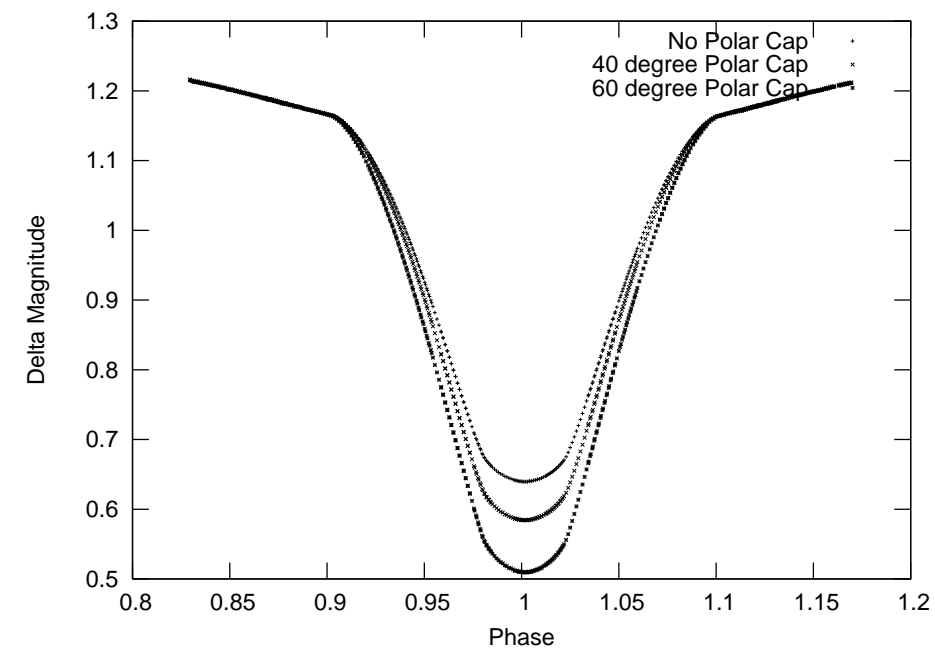

Fig. 9.- Variation of a theoretical primary eclipse lightcurve due to the presence of a polar spot of radius of $40^{\circ}$ and $60^{\circ}$. For comparison the primary eclipse lightcurve without a polar spot is also plotted. We show only the primary eclipse as this is the part of the lightcurve that is most effected by the presence of a polar cap.

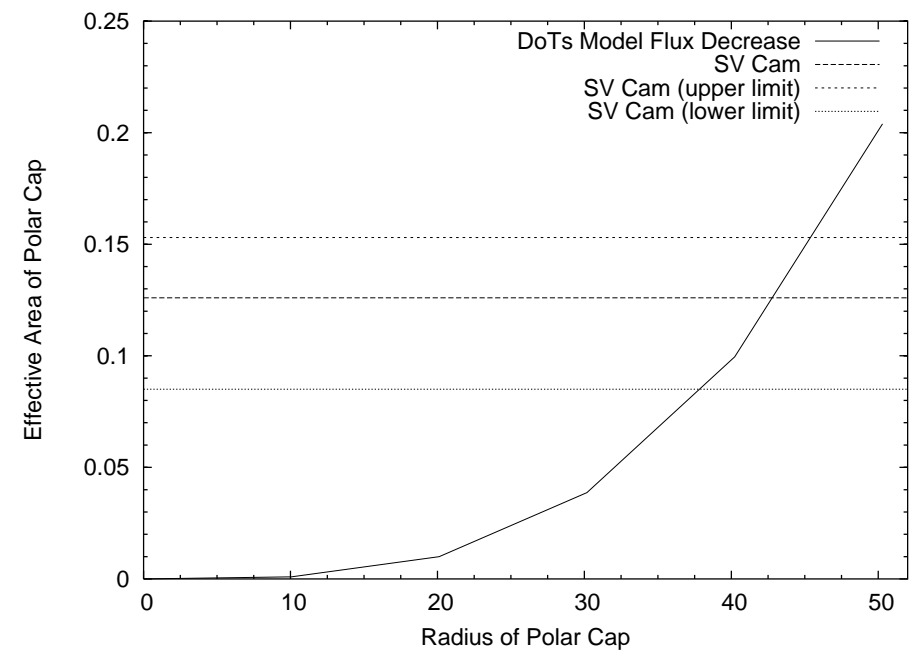

Fig. 10. - Plot of the fractional decrease in the stellar flux of SV Cam as a function of theoretical polar spot size radius in degrees. Also plotted is the fractional missing light for SV Cam as determined in the previous section. 


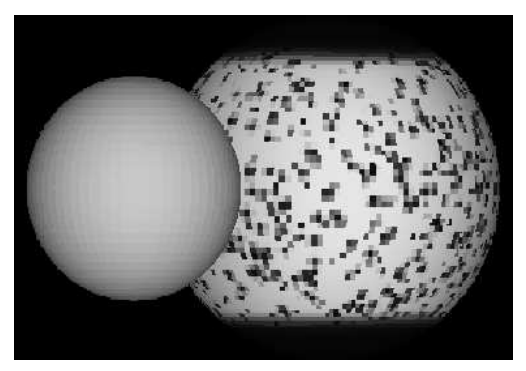

Fig. 11.- A model of SV Cam showing $28 \%$ spot coverage and a $42^{\circ}$ polar cap.

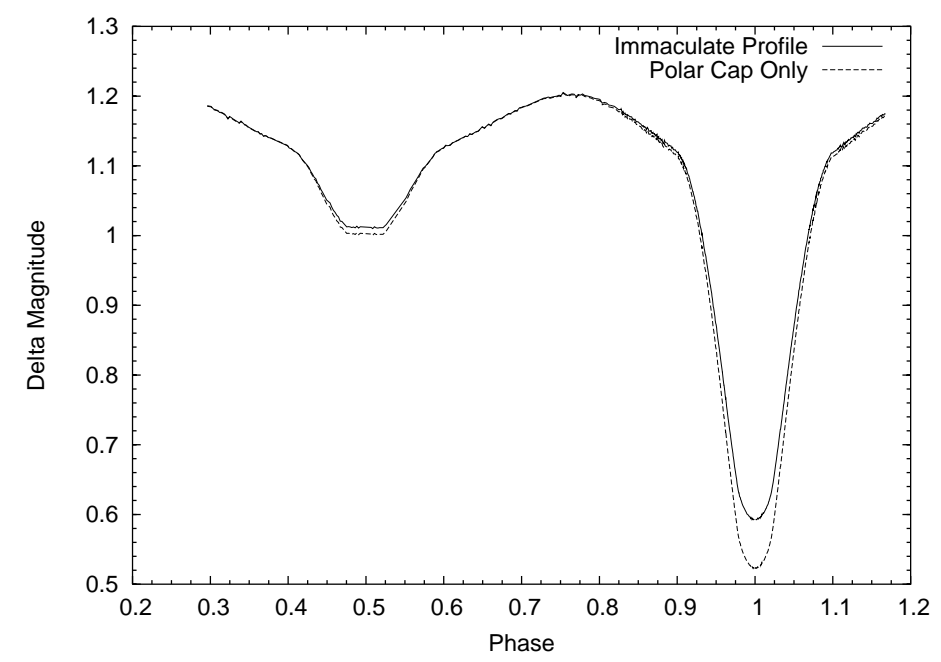

Fig. 12. - The effect of a $42^{\circ}$ polar cap on the lightcurve of SV Cam. For comparison an immaculate profile, with no star-spots, is also plotted.

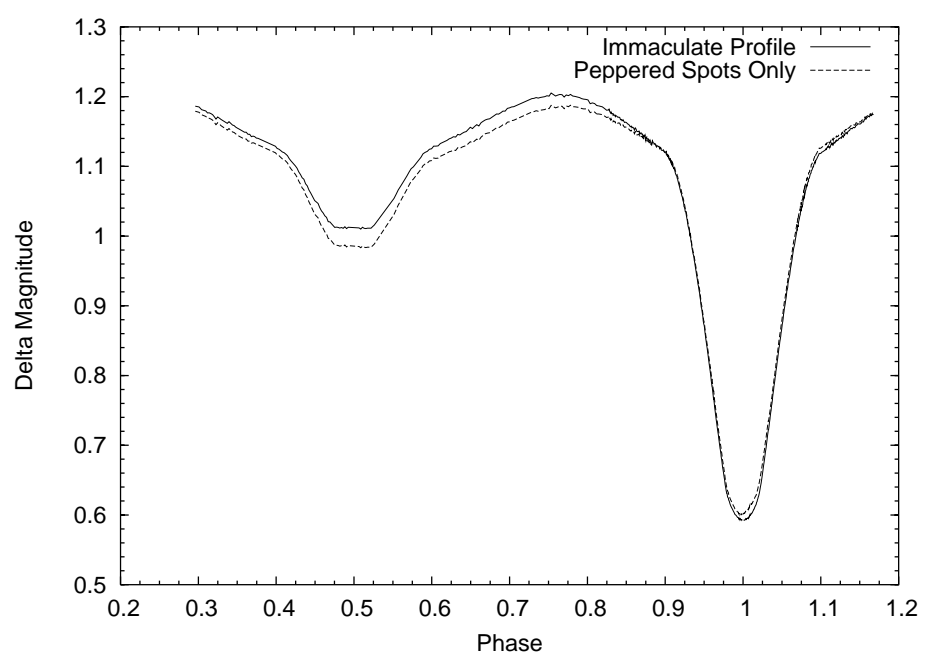

Fig. 13. - The effect of $28 \%$ small star-spot coverage on SV Cam. For comparison an immaculate profile, with no star-spots, is also plotted. 


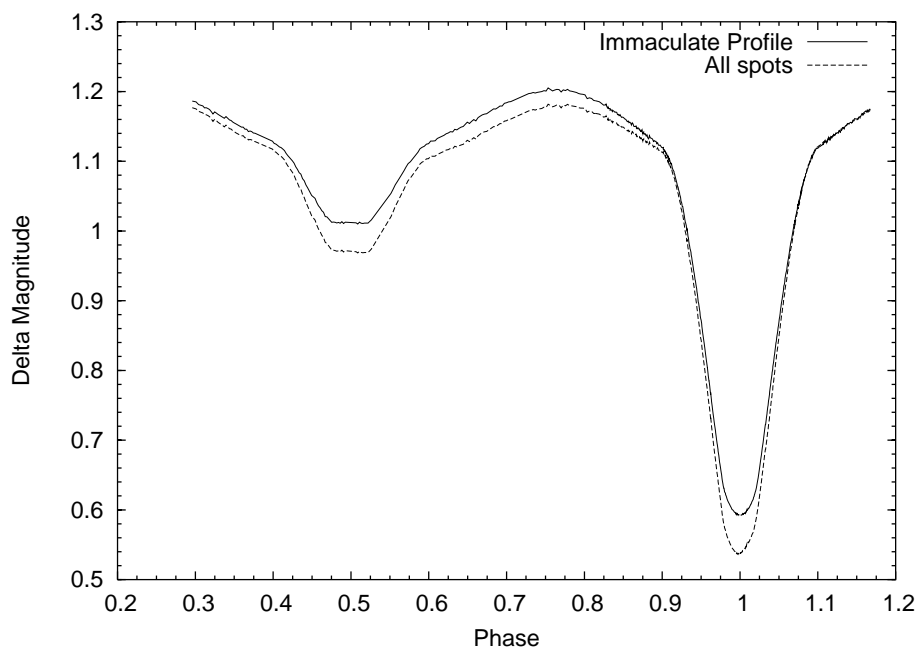

Fig. 14. - The effect of a combination of $42^{\circ}$ polar cap and $28 \%$ spot coverage on the lightcurve of SV Cam. For comparison an immaculate profile, with no star-spots, is also plotted. 


\begin{tabular}{lccccc}
\hline \hline Visit & Obs. Date & UT & UT & Exposure & No of \\
& & Start & End & $\begin{array}{c}\text { Time(s) } \\
\text { Frames }\end{array}$ \\
\hline 1 & 01 November 2001 & $20: 55: 56$ & $01: 00: 17$ & 30 & 165 \\
2 & 03 November 2001 & $14: 34: 29$ & $18: 38: 21$ & 30 & 165 \\
3 & 05 November 2001 & $09: 49: 17$ & $13: 52: 22$ & 30 & 165 \\
\hline \hline
\end{tabular}

Table 1: HST Observations of SV Cam 


\begin{tabular}{lcc}
\hline \hline & Primary Star & Secondary Star \\
\hline Radius & $1.24 \pm 0.04 \mathrm{R}_{\odot}$ & $0.79 \pm 0.03 \mathrm{R}_{\odot}$ \\
Mass & $1.14 \pm 0.03 \mathrm{M}_{\odot}$ & $0.73 \pm 0.02 \mathrm{M}_{\odot}$ \\
$\log \mathrm{g}$ & $4.31 \pm 0.04$ & $4.5 \pm 0.03$ \\
\hline \hline
\end{tabular}

Table 2: Stellar Parameters of SV Cam 